\title{
Differential roles of Scavenger receptor class B type I: A protective molecule and a facilitator of atherosclerosis (Review)
}

\author{
BAITAO MA ${ }^{1}$, JING JIA $^{2}$, XUEBIN WANG $^{1}$, RUI ZHANG ${ }^{1}$, SHUAI NIU $^{1}$, \\ LENG NI $^{1}, \mathrm{XIAO} \mathrm{DI}^{1}$ and CHANGWEI LIU ${ }^{1}$ \\ ${ }^{1}$ Department of Vascular Surgery, Peking Union Medical College Hospital, Chinese Academy of Medical Sciences and \\ Peking Union Medical College, Beijing 100730; ${ }^{2}$ Department of Obstetrics and Gynaecology, Tongji Hospital of \\ Tongji Medical College, Huazhong University of Science and Technology, Wuhan, Hubei 430030, P.R. China
}

Received April 15, 2020; Accepted July 3, 2020

DOI: $10.3892 / \mathrm{mmr} .2020 .11383$

\begin{abstract}
The scavenger receptor class B type I (SR-BI) is a multi-ligand membrane protein receptor that binds to high-density lipoprotein (HDL) under physiological conditions, promoting the selective uptake of cholesterol esters from HDL into cells. SR-BI also promotes the reverse transport of excess cholesterol from peripheral tissues to the liver, contributing to the synthesis of bile acids for excretion and the removal of excess cholesterol from the body, thereby lowering the cholesterol load and exerting anti-atherosclerotic effects. Studies in mice and humans have demonstrated that a functional defect of SR-BI can cause atherosclerotic lesions and cardiovascular diseases, such as myocardial infarction and stroke. Additionally, SR-BI in vascular endothelial cells promoted the deposition of low-density lipoprotein under the endothelium. Although SR-BI is widely expressed in various tissues and cell types throughout the body, its expression level and function vary accordingly. The present review focuses on the biological functions and mechanisms of SR-BI in regulating atherosclerosis.
\end{abstract}

Correspondence to: Professor Changwei Liu, Department of Vascular Surgery, Peking Union Medical College Hospital, Chinese Academy of Medical Sciences and Peking Union Medical College, 1 Shuaifuyuan Hutong, Dongcheng, Beijing 100730, P.R. China E-mail: liucw@vip.sina.com

Abbreviations: SR-BI, scavenger receptor class B type I; HDL, high-density lipoprotein; EC, endothelial cell; LIMP-2, lysosomal integral membrane protein 2; CE, cholesterol ester; LDL, low-density lipoprotein; VLDL, very-low-density lipoprotein; Lpa, lipoprotein a; PS, phosphatidylserine; LDLR, LDL receptor; FC, free cholesterol; RCT, reverse cholesterol transport; DOCK4, dedicator of cytokinesis 4; ApoE, apolipoprotein E; TC, total cholesterol; BMDC, bone marrow-derived cell

Key words: atherosclerosis, cardiovascular disease, scavenger receptor class B type I, high-density lipoprotein, low-density lipoprotein

\section{Contents}

1. Introduction

2. Structure of SR-BI

3. SR-BI in hepatocytes

4. SR-BI in endothelial cells

5. SR-BI in macrophages

6. SR-BI in platelets

7. Conclusion

\section{Introduction}

The gene sequence of scavenger receptor class B type I (SR-BI) was first determined by Calvo and Vega (1) in 1993 and named CLA-1 (now SCARBI). SR-BI shares close sequence identity with cluster determinant 36 and lysosomal integral membrane protein 2 (LIMP-2). Acton et al (2) proposed that SR-BI was a high-affinity high-density lipoprotein (HDL) physiological membrane protein receptor that mediated the selective uptake of cholesterol esters (CEs) in HDL particles via hepatocytes. Subsequent research has focused on the transcriptional regulation, structure and function of SR-BI. SR-BI is highly conserved across different species, and is widely expressed in different tissues, including liver, gonad, adrenal gland, intestinal tract, macrophages, endothelial cells (ECs), platelets, placenta, adipocytes and smooth muscle cells (3). SR-BI is a member of the scavenger receptor superfamily and can recognize a variety of ligands, including HDL, natural and modified low-density lipoprotein (LDL), very-low-density lipoprotein (VLDL), anion-containing phospholipids, lipoprotein a (Lpa), phosphatidylserine (PS) and apoptotic cells (4). SR-B1 has also been demonstrated to exhibit a high affinity for native $\operatorname{HDL}(4,5)$.

The main physiological function of SR-BI is binding with HDL to mediate the selective uptake and reverse transport of cholesterol (3). When circulating LDL particles bind to the LDL receptor (LDLR), cholesterol is delivered to cells via endocytosis of the holo-particle (3). After endocytosis, CEs carried in LDL are hydrolysed by acid lipase in the lysosome, releasing free cholesterol (FC) that is transported to the endoplasmic reticulum, where it is either re-esterified to CEs and stored in lipid droplets in the cytoplasm, or transported to the 
plasma membrane (3). In contrast, the mechanism by which CEs in HDL particles enter the cell is completely different from internalisation of the CE-rich lipoprotein particles, which is mediated by LDLR. After HDL particles bind to SR-BI on the cell surface, CEs, FC and triglycerides are selectively delivered to the inside of the cell through hydrophobic channels formed by the extracellular domain of the SR-BI molecule, while the whole lipoprotein particle remains outside the cell $(2,6)$. This process results in the selective uptake of cholesterol. In steroidogenic cells, CEs that enter the cell in this way are hydrolysed by hormone-sensitive lipases before being used (7).

HDL is an anti-atherosclerotic lipoprotein, possibly due to its role in reverse cholesterol transport (RCT) (6). A meta-analysis indicated that a decrease in plasma HDL was associated with increased occurrence of atherosclerosis and cardiovascular diseases (8). Excess cholesterol in macrophages or foam cells in atherosclerotic lesions can be transferred to HDL particles through unidirectional outflow mediated by ATP-binding cassette A1 and ATP-binding cassette G1 (9), or flow to mature HDL particles via passive diffusion through hydrophobic channels formed by the extracellular domain of SR-B1 molecules (9). Both mechanisms decrease the storage of CEs in cells and reduce the incidence of atherosclerosis and the risk of cardiovascular diseases (9). Mature HDL particles circulate through the blood to the liver, where they interact with SR-BI on the hepatocyte's membrane (10). This reverse transport of cholesterol to the liver results in the synthesis of bile acids for secretion into the lumen of the intestine and the ensuing excretion of excess cholesterol from the body (10). Interactions between HDL and SR-BI on the hepatocyte membrane and the selective uptake of CEs are crucial RCT steps, which serve a critical role in cardiovascular protection (11).

\section{Structure of SR-BI}

The human SCARBI gene encoding the SR-BI protein is located at $12 \mathrm{q} 24$ on chromosome 12 , is $\sim 87 \mathrm{~kb}$ in length and comprises 13 exons and 12 introns (3). SR-B1 has a predicted molecular weight of $57 \mathrm{kDa}$ and contains multiple glycosylation sites (3). However, due to post-translational modification, it migrates at $\sim 82 \mathrm{kDa}$ when assessed using SDS-PAGE (12).

The human $S C A R B 1 \mathrm{mRNA}$ yields five protein variants through alternative splicing (3). Isoform 1 is the main gene product, consisting of 509 amino acid (aa) residues from exons 1-12. This was the first isoform identified and named SR-BI (3). Isoform 2 comprises 409 aa residues and has the same C-terminus as isoform 1 but lacks aa residues 43-142. Isoform 3 is the longest variant with 552 aa residues. Isoform 4 (also called SR-BIII) is a polypeptide comprised of 474 aa, with the same C-terminus as isoform 1 , but a different $\mathrm{N}$-terminus (aa 1-42). Isoform 5 (also called SR-BII) comprises 506 aa residues and has a unique $\mathrm{C}$-terminus (aa 468-552).

SR-B1 has two short intracellular domains at the $\mathrm{N}$-terminus (aa 1-11) and C-terminus (aa 462-509), two transmembrane domains (aa 12-32 and 441-461) and a large extracellular domain containing multiple N-linked glycosylation sites (3). The C-terminal cytoplasmic domain is essential for SR-BI signal transduction and contains kinase phosphorylation sites and protein binding sites, as well as a serine that can be phosphorylated by the kinase SIK-1, which increases the efficiency of CEs uptake mediated by SR-BI (3).
A common C-terminal sequence (aa 468-552) in isomers 1, 2 and 4 includes the VLQEAKL motif that is required for binding to the PDZ domain-containing protein PDZK1, which is essential for maintaining the stability and function of SR-BI (13). The other C-terminal sequence (aa 487-494), IQAYSESL, is a motif required to bind the guanine nucleotide exchange factor dedicator of cytokinesis 4 (DOCK4), which is essential for the uptake and transendocytosis of LDL in ECs (14). Determination of the crystal structure of LIMP-2, a homologue of SR-B1, provided a basic framework for the structure of this protein family (15), which revealed that the main extracellular domain of SR-BI contained an antiparallel $\beta$-barrel core and multiple short $\alpha$-helical structures that facilitated the formation of a hydrophobic channel and promoted cholesterol transport.

\section{SR-BI in hepatocytes}

SR-BI on the hepatocyte membrane mediates the selective uptake of CEs in plasma HDL particles and the RCT process. SR-B1 is also known to regulate lipoprotein homeostasis, inhibit the development of atherosclerosis and reduce the incidence of cardiovascular diseases $(16,17)$.

Van Eck et al (18) established SR-BI knockout (SR-BI ${ }^{--}$) mice and fed them a western-type diet for 20 weeks. Compared with wild-type mice, cholesterol levels in the liver and the expression of key regulatory factors related to cholesterol homeostasis [3-hydroxy-3-methyl-glutaryl-coenzyme A (HMG-CoA) reductase, LDLR and cholesterol $7 \alpha$-hydroxylase] were not affected, but a large amount of cholesterol-rich HDL accumulated in the circulatory system along with abnormally large HDL particles, which indicated impaired cholesterol delivery to the liver. SR-BI-deficient mice displayed an $\sim 40 \%$ reduction in biliary cholesterol content, lipid deposition in the aortic wall and the increased occurrence of atherosclerosis, which indicated that the RCT process was damaged and the cholesterol clearance capacity was impaired (18). Apolipoprotein E (ApoE) single-knockout mice did not usually suffer early atherosclerosis, severe cardiac dysfunction, cardiac infarction or premature death when fed a standard diet. However, SR-BI and ApoE double gene knockout mice experienced severe hypercholesterolemia after ingesting a standard diet, and atherosclerotic lesions appeared in the early stages (4-5 weeks), resulting in coronary artery occlusion, myocardial infarction and cardiac dysfunction, with most mice dying at 5-8 weeks of age $(19,20)$. Moreover, after a period of high-fat and high-cholesterol consumption, SR-BI and LDLR double gene knockout mice exhibited higher cholesterol expression levels, more severe atherosclerotic lesions and a higher incidence of myocardial infarction and mortality, compared with LDLR knockout (LDLR $\left.{ }^{-/}\right)$mice in the control group (21). These studies indicated that SR-BI serves an important role in the prevention of atherosclerosis and the reduction of cardiovascular diseases, partly due to selective cholesterol uptake and RCT mediated by SR-BI on the hepatocyte membrane.

SR-BI on hepatocytes is beneficial to cardiovascular fitness through the regulation of HDL cholesterol metabolism (22). Liver-specific SR-BI deficiency induced atherosclerotic lesions, while overexpression of SR-BI in hepatocytes decreased atherosclerosis (22). After transient overexpression of SR-BI in hepatocytes, $\mathrm{LDLR}^{-/-}$mice fed with a high-fatandhigh-cholesterol 
diet displayed a marked reduction in early and advanced atherosclerotic lesions, and also experienced significantly decreased HDL cholesterol but unchanged or only modestly reduced non-HDL cholesterol levels (23). Huby et al (24), produced a hypomorphic allele (hypomSR-BI), an SR-BI conditional knockout mouse model, by targeting the SR-BI gene through loxP site insertion, resulting in a decrease in SR-BI expression. After consuming an atherogenic diet for 11 weeks, plasma total cholesterol (TC) levels were increased 2-fold compared with the control group, and hypomSR-BI-KO (liver) and SR-BI ${ }^{-/}$mice developed severe hypercholesterolemia due to the accumulation of VLDL-sized particles in the circulation (24). The degree of atherosclerosis was more severe in hypomSR-BI mice, hypomSR-BI-KO (liver) mice and SR-BI ${ }^{-/-}$mice compared with the control mice, by 2.5-fold, 32-fold and 48-fold, respectively. There was no difference in plasma lipid expression levels between hypomSR-BI-KO (liver) mice and $\mathrm{SR}_{-} \mathrm{BI}^{-/}$mice. However, compared with SR-BI ${ }^{-/}$mice, hypomSR-BI-KO (liver) mice had fewer atherosclerotic lesions (24). The aforementioned studies indicated that SR-BI expressed in hepatocytes serves a critical role in anti-atherosclerosis, and SR-BI expressed in other tissues or cells also perform anti-atherosclerotic functions to a limited extent.

In addition to the selective uptake of CEs from HDL, SR-BI expressed in the liver can also clear VLDL and Lpa. Van Eck et al (25) demonstrated that at 20 mins after the intravenous injection of (125)I- $\beta$-VLDL, the liver uptake ability of SR-BI ${ }^{-/-}$mice was decreased by $44 \%$ compared with that of wild-type mice, while VLDL in plasma was significantly increased $(45.9 \pm 8.8$ vs. $6.1 \pm 2.3 \mu \mathrm{g} / \mathrm{ml})$. A study by Yang et al (26) demonstrated that SR-BI acted as a receptor for atherosclerotic particles containing Lpa and mediated the selective uptake of lipids in Lpa. SR-BI ${ }^{-/-}$mice presented with the significantly reduced clearance rate of Lpa in the plasma, while SR-BI overexpression in the liver could significantly increase the clearance rate. Humans carry a number of different $S C A R B 1$ gene mutations that reduce the ability of the liver to uptake CEs in HDL and Lpa, resulting in significantly elevated plasma HDL and Lpa expression levels (27), although this is accompanied by higher HDL levels and the risk of cardiovascular disease is significantly increased. The aforementioned studies in mice and humans indicated that in addition to mediating the reverse transport of cholesterol in surrounding tissues to liver cells via HDL particles, generating and excreting bile, liver SR-BI also had an anti-atherogenic role via regulating the homeostasis of lipoproteins in the blood.

\section{SR-BI in endothelial cells}

SR-BI in ECs not only binds with HDL to activate downstream signalling pathways (28) or transport HDL to the subintimal region via transcytosis (29) to exert an anti-atherosclerotic effect, but also mediates the delivery of LDL into the artery wall via transcytosis $(14,30)$, thereby promoting atherosclerosis (Fig. 1).

SR-BI regulates the function of ECs under the stimulation of HDL via activating Src kinases, phosphatidylinositol 3-kinase $(\mathrm{PI} 3 \mathrm{~K})$, protein kinase $\mathrm{B} / \mathrm{mitogen}$-activated protein kinases and the Rac GTP enzyme, resulting in changes in the actin cytoskeleton, promotion of EC migration (Fig. 1), enhancement of EC repair and regeneration and improvement of endothelial monolayer integrity in vivo $(31,32)$. The HDL/SR-BI interaction induced the phosphorylation of endothelial nitric oxide synthase and the synthesis of nitric oxide via phosphorylation of Src kinases, protected ECs from apoptosis and promoted the growth and migration of ECs (32). Moreover, phosphorylation of Src kinases induced by HDL/SR-BI required the assistance of the adaptor protein PDZK1 $(3,33)$. In the aforementioned study, compared with the control group, EC-specific SR-BI-overexpressing $\mathrm{C} 57 \mathrm{BL} / 6 \mathrm{~N}$ mice and $\mathrm{ApoE}^{-/}$mice fed a high-fat and high-cholesterol diet displayed significantly decreased plasma levels of TC and FC, while HDL was remarkably increased, and the severity of atherosclerosis was decreased. However, for C57BL/6N mice and $\mathrm{ApoE}^{-/-}$mice fed a normal chow diet, EC-specific SR-BI overexpression had no significant effect on plasma lipids and lipoproteins (34), suggesting that SR-BI expressed on ECs may serve an important role in the regulation of lipid and lipoprotein homeostasis and preventing atherosclerosis.

SR-BI on ECs also serve a role in transcytosis, transporting plasma lipoproteins to the endometrium $(29,30)$. Rohrer et al (29) indicated that SR-BI could mediate the transport of HDL in the circulation to the intima of vessels, receive cholesterol in foam cells or macrophages and reduce cholesterol deposition beneath the arterial endothelium, thereby exerting an anti-atherosclerotic effect. The diameter of translocated HDL particles was decreased, but protein moieties remained intact (29). A study by Fung et al (35) demonstrated that SR-BI on the brain microvascular endothelium could mediate HDL transcytosis, and this process did not depend on the adaptor protein PDZK1. Armstrong et al (30) revealed that SR-BI mediated the transcytosis of LDL in ECs. Overexpression of SR-BI enhanced the endocytosis of LDL in ECs, but the effect was significantly reduced when an excess of HDL (a high-affinity ligand for SR-BI) was administered or the expression of SR-BI was depleted by small interfering RNA-mediated silencing (30).

A recent study by Huang et al (14) demonstrated that SR-BI mediated LDL transcytosis associated with ECs via DOCK4 to promote the development of atherosclerosis (Fig. 1). Transcytosis of LDL particles through ECs required direct binding to SR-BI expressed on the apical membranes of ECs, and an 8 aa (IQAYSESL) cytoplasmic motif in C-terminus of SR-BI that recruited DOCK4 (36), which promoted the internalisation of SR-BI and transcytosis of LDL across EC monolayers by coupling LDL and SR-BI via Rho GTPases Ras-associated C3 botulinum toxin substrate 1 (Rac1) activation (14). Selective deletion of SR-BI in ECs in male and female wild-type, $\mathrm{ApoE}^{-/-}$and $\mathrm{LDLR}^{-/-}$mice had the protective effect against atherosclerosis but it did not alter circulating levels of TC, HDL or the lipoprotein profile (14). The aforementioned results indicated that the role of SR-BI expressed on ECs in atherosclerosis is complex, and diverse results from different studies might be related to the metabolic state of the body and the cholesterol load, which suggested that accurate individualised treatment in different populations could be achieved when formulating anti-atherosclerosis strategies targeting SR-BI.

\section{SR-BI in macrophages}

The SR-BI protein was also expressed on macrophages, such as Kupffer cells in the liver (37) and foam cells in atherosclerotic plaques (38). SR-BI expressed on macrophages has an important 


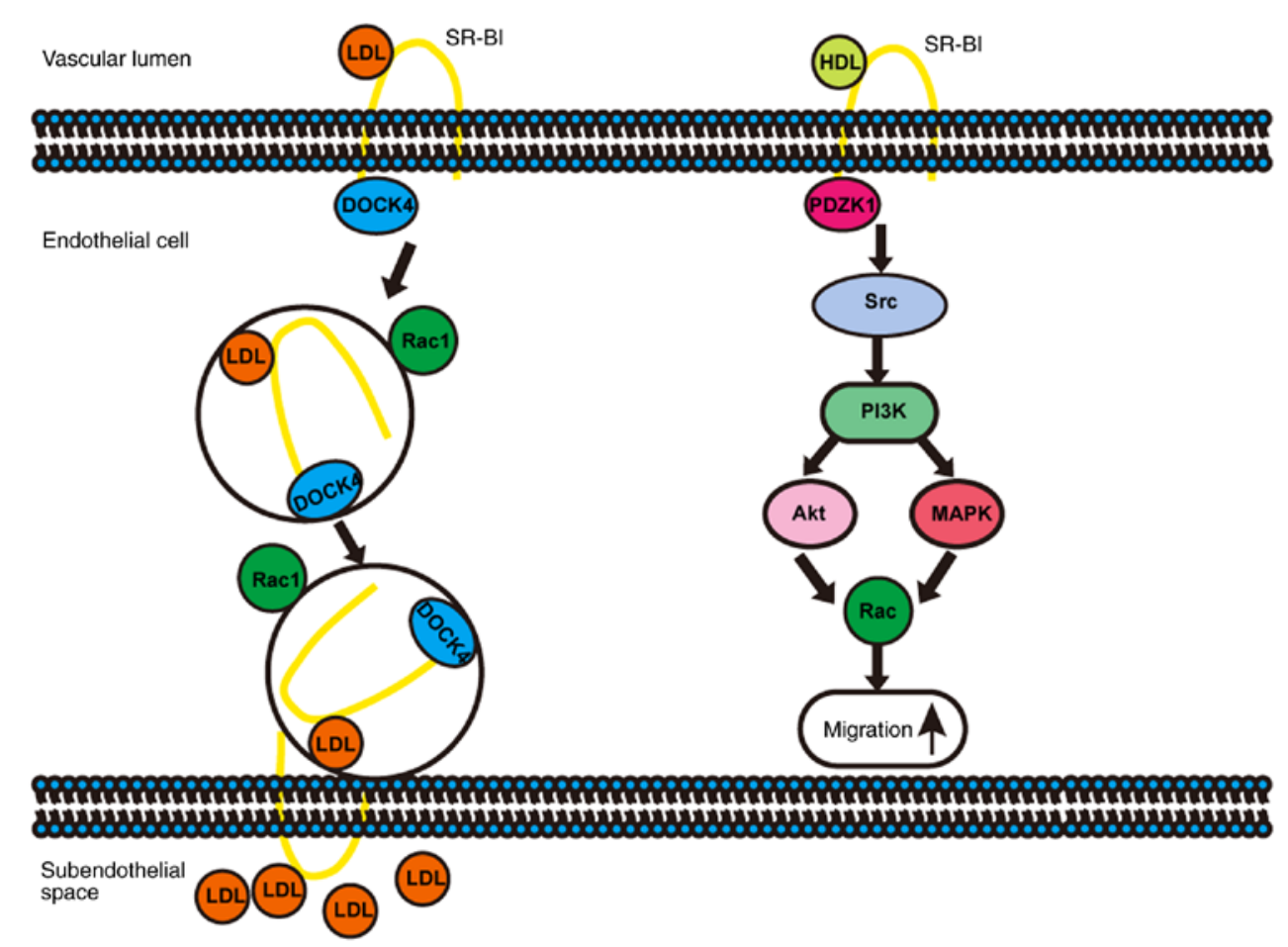

Figure 1. Schematic. SR-BI promotes transcytosis of LDL and migration of ECs. SR-BI, scavenger receptor class B type I; HDL, high-density lipoprotein; LDL, low-density lipoprotein; EC, endothelial cell; DOCK4, dedicator of cytokinesis 4; PDZK1, PDZ domain-containing 1.

role in selective cholesterol uptake (39), cholesterol efflux $(40,41)$, HDL-induced anti-inflammatory responses $(41,42)$ and the inhibition of atherosclerotic progression (43).

Tao et al (43) demonstrated that macrophage SR-BI bound to PS and induced Src phosphorylation, which led to the activation of the downstream molecules PI3K and Rac1, phagocytosis and elimination of apoptotic cells in atherosclerotic lesions, thereby reducing necrosis, inhibiting inflammatory responses and exerting an anti-atherosclerotic effect. Moreover, SR-BI also mediated HDL-induced anti-inflammatory effects by inhibiting the activation of $\mathrm{NF}-\kappa \mathrm{B}$ and the production of tumor necrosis factor $\alpha(42)$.

Although SR-BI was expressed in many bone marrow-derived cells (BMDCs), including B and T lymphocytes, lymphocyte SR-BI did not affect the development of atherosclerosis (44). In order to investigate the role of macrophage SR-BI in atherosclerosis, Van Eck et al (45) used bone marrow transplantation technology to specifically regulate the expression of SR-BI in leukocytes of $\mathrm{LDLR}^{-/-}$mice, and indicated that macrophage SR-BI had a unique dual role in the process of atherosclerosis. After feeding LDLR $^{-/}$mice with a western-type diet for 9 or 12 weeks, the presence of SR-BI in BMDCs reduced the development of atherosclerosis. However, after only 4 weeks in $\mathrm{LDLR}^{-/-}$mice fed a western diet and wild-type mice fed a high-cholesterol and high-cholate diet, expression of SR-BI in BMDCs promoted the development of small fat streak lesions (45). In addition, SR-BI-null and apoE-hypomorphic $\left(\mathrm{SR}_{-} \mathrm{BI}^{-/-} / \mathrm{ApoE}^{+/}\right)$mice transplanted with bone marrow derived from $\mathrm{SR}-\mathrm{BI}^{+/ /} / \mathrm{ApoE}^{+/-}$mice and fed a high-fat high-cholesterol and cholate-containing diet for 4 weeks revealed a significant reduction in the degree of coronary atherosclerosis and the incidence of myocardial infarction compared with mice transplanted with bone marrow derived from SR-BI ${ }^{-/-}$mice (46).
SR-BI expressed by macrophages has also been revealed to mediate cholesterol efflux from macrophages into mature HDL particles (40), which is an important stage in the process of anti-atherosclerosis. Compared with the wild-type group, the rate of cholesterol efflux from macrophages isolated from $\mathrm{SR}-\mathrm{BI}^{-/-}$mice to mature $\mathrm{HDL}$ particles was reduced by $20 \%$. In addition, Badeau et al (47) indicated that SR-BI inhibitors significantly reduced cholesterol efflux from THP-1 macrophages. A previous clinical study has demonstrated that cholesterol outflow to HDL in macrophages from human carriers of the SCARB1 gene P297S mutation was significantly reduced compared with control subjects (48). In conclusion, macrophage SR-BI exerted an anti-atherosclerotic effect by eliminating apoptotic cells, inducing anti-inflammatory responses and promoting cholesterol efflux.

\section{SR-BI in platelets}

Platelets are closely associated with the development of atherosclerosis (49), and their activation and aggregation can generate thrombus, accelerate the progression of atherosclerotic lesions and subsequently occlude arteries, which results in reduced blood/oxygen supply to organs or tissues, ultimately leading to the occurrence of cardiovascular events, such as myocardial infarction and stroke $(49,50)$. Therefore, inhibiting the abnormal aggregation and activation of platelets could reduce the occurrence of cardiovascular events (50). Valiyaveettil et al (51) found that the SR-BI protein was present in both resting and activated platelets, and that oxidised HDL particles could bind to and interact with SR-BI to inhibit platelet activation and aggregation, thereby reducing the risk of thrombosis. In addition, Brodde et al (52) demonstrated that natural $\mathrm{HDL}_{3}$ (the main component of HDL) bound to 
SR-BI on platelets and activated the signal pathways, which inhibited platelet activation. Furthermore, $\mathrm{SR}-\mathrm{BI}^{-/}$mice were more likely to suffer deep vein thrombosis (53), and the time required for arterial occlusion was shorter in a model of $\mathrm{FeCl}_{3}$-induced arterial thrombosis (54). The increase of circulating platelet and bone marrow megakaryocyte numbers was impaired in $\mathrm{SR}-\mathrm{BI}^{-/}$mice when challenged with thrombopoietin. However, proplatelets were normally produced by megakaryocytes from normolipidemic bone marrow-specific $\mathrm{SR}_{-\mathrm{BI}}{ }^{-/}$mice (55). Furthermore, studies in humans indicated that carriers of the P297S mutation of the SCARB1 gene had significantly increased FC levels in platelets and impaired platelet function (48).

\section{Conclusion}

In conclusion, SR-BI is a multi-ligand membrane receptor that has a high affinity for HDL and is believed to be the physiological receptor for HDL. Expression of SR-BI in different tissues and cell types exhibit different roles in the process of atherosclerosis. SR-BI in hepatocytes and platelets have been demonstrated to inhibit the development of atherosclerotic lesions and thrombosis, and act as a protective factor for cardiovascular diseases $(16,17)$. However, SR-BI expressed in vascular ECs has been indicated to promote the deposition of LDL under the endothelium, aggravating atherosclerosis and representing a risk factor for cardiovascular diseases. The effects of macrophage SR-BI on the pathogenesis of atherosclerosis and cardiovascular diseases are complex and depend on the metabolic and inflammatory status at different stages of atherosclerosis. Therefore, the role of SR-BI in the cardiovascular system remains ambiguous, and all the previously reported cardioprotective and atherosclerotic effects of SR-BI must be considered when designing therapeutic agents to treat atherosclerosis and cardiovascular diseases.

\section{Acknowledgements}

The authors would like to thank their colleagues, Dr Yunfei Chen and Dr Chunling Xue (Medical Science Research Center, Chinese Academy of Medical Sciences and Peking Union Medical College) for their helpful support and editing of this manuscript.

\section{Funding}

The present study was supported by the National Natural Science Foundation of China (grant no. 81970417) and the Fundamental Research Funds for the Central Universities (grant no. 3332019028).

\section{Availability of data and materials}

Not applicable.

\section{Authors' contributions}

BTM and JJ wrote the manuscript. XBW, RZ, SN, LN, XD and CWL reviewed and edited the manuscript. All authors read and approved the final manuscript.

\section{Ethics approval and consent to participate}

Not applicable.

\section{Patient consent for publication}

Not applicable.

\section{Competing interests}

The authors declare that they have no competing interests.

\section{References}

1. Calvo D and Vega MA: Identification, primary structure, and distribution of CLA-1, a novel member of the CD36/LIMPII gene family. J Biol Chem 268: 18929-18935, 1993.

2. Acton S, Rigotti A, Landschulz KT, Xu S, Hobbs $\mathrm{HH}$ and Krieger M: Identification of scavenger receptor SR-BI as a high density lipoprotein receptor. Science 271: 518-520, 1996.

3. Shen WJ, Azhar S and Kraemer FB: SR-B1: A unique multifunctional receptor for cholesterol influx and efflux. Annu Rev Physiol 80: 95-116, 2018.

4. Shen WJ, Asthana S, Kraemer FB and Azhar S: Scavenger receptor B type 1: Expression, molecular regulation, and cholesterol transport function. J Lipid Res 59: 1114-1131, 2018.

5. Shen WJ, Hu J, Hu Z, Kraemer FB and Azhar S: Scavenger receptor class B type I (SR-BI): A versatile receptor with multiple functions and actions. Metabolism 63: 875-886, 2014.

6. Hoekstra M: SR-BI as target in atherosclerosis and cardiovascular disease-A comprehensive appraisal of the cellular functions of SR-BI in physiology and disease. Atherosclerosis 258: 153-161, 2017.

7. Kraemer FB, Shen WJ, Harada K, Patel S, Osuga J, Ishibashi S and Azhar S: Hormone-sensitive lipase is required for high-density lipoprotein cholesteryl ester-supported adrenal steroidogenesis. Mol Endocrinol 18: 549-557, 2004.

8. Prospective Studies Collaboration, Lewington S, Whitlock G, Clarke R, Sherliker P, Emberson J, Halsey J, Qizilbash N, Peto R and Collins R: Blood cholesterol and vascular mortality by age, sex, and blood pressure: A meta-analysis of individual data from 61 prospective studies with 55,000 vascular deaths. Lancet 370: 1829-1839, 2007.

9. Phillips MC: Molecular mechanisms of cellular cholesterol efflux. J Biol Chem 289: 24020-24029, 2014

10. Ji Y, Wang N, Ramakrishnan R, Sehayek E, Huszar D, Breslow JL and Tall AR: Hepatic scavenger receptor BI promotes rapid clearance of high density lipoprotein free cholesterol and its transport into bile. J Biol Chem 274: 33398-33402, 1999.

11. Brundert M, Ewert A, Heeren J, Behrendt B, Ramakrishnan R, Greten H, Merkel M and Rinninger F: Scavenger receptor class B type I mediates the selective uptake of high-density lipoprotein-associated cholesteryl ester by the liver in mice. Arterioscler Thromb Vasc Biol 25: 143-148, 2005.

12. Acton SL, Scherer PE, Lodish HF and Krieger M: Expression cloning of SR-BI, a CD36-related class B scavenger receptor. J Biol Chem 269: 21003-21009, 1994.

13. Trigatti BL: SR-B1 and PDZK1: Partners in HDL regulation. Curr Opin Lipidol 28: 201-208, 2017.

14. Huang L, Chambliss KL, Gao X, Yuhanna IS, Behling-Kelly E, Bergaya S, Ahmed M, Michaely P, Luby-Phelps K, Darehshouri A, et al: SR-B1 drives endothelial cell LDL transcytosis via DOCK4 to promote atherosclerosis. Nature 569: 565-569, 2019.

15. Neculai D, Schwake M, Ravichandran M, Zunke F, Collins RF, Peters J, Neculai M, Plumb J, Loppnau P, Pizarro JC, et al: Structure of LIMP-2 provides functional insights with implications for SR-BI and CD36. Nature 504: 172-176, 2013.

16. Linton MF, Tao H, Linton EF and Yancey PG: SR-BI: A multifunctional receptor in cholesterol homeostasis and atherosclerosis. Trends Endocrinol Metab 28: 461-472, 2017.

17. Sahebi R, Hassanian SM, Ghayour-Mobarhan M, Farrokhi E, Rezayi M, Samadi S, Bahramian S, Ferns GA and Avan A: Scavenger receptor Class B type I as a potential risk stratification biomarker and therapeutic target in cardiovascular disease. J Cell Physiol 234: 16925-16932, 2019. 
18. Van Eck M, Twisk J, Hoekstra M, Van Rij BT, Van der Lans CA, Bos IS, Kruijt JK, Kuipers F and Van Berkel TJ: Differential effects of scavenger receptor BI deficiency on lipid metabolism in cells of the arterial wall and in the liver. J Biol Chem 278: 23699-23705, 2003.

19. Braun A, Trigatti BL, Post MJ, Sato K, Simons M, Edelberg JM, Rosenberg RD, Schrenzel M and Krieger M: Loss of SR-BI expression leads to the early onset of occlusive atherosclerotic coronary artery disease, spontaneous myocardial infarctions, severe cardiac dysfunction, and premature death in apolipoprotein E-deficient mice. Circ Res 90: 270-276, 2002.

20. Braun A, Zhang S, Miettinen HE, Ebrahim S, Holm TM, Vasile E, Post MJ, Yoerger DM, Picard MH, Krieger JL, et al: Probucol prevents early coronary heart disease and death in the high-density lipoprotein receptor SR-BI/apolipoprotein E double knockout mouse. Proc Natl Acad Sci USA 100: 7283-7288, 2003

21. Fuller M, Dadoo O, Serkis V, Abutouk D, MacDonald M, Dhingani N, Macri J, Igdoura SA and Trigatti BL: The effects of diet on occlusive coronary artery atherosclerosis and myocardial infarction in scavenger receptor class B, type $1 /$ low-density lipoprotein receptor double knockout mice. Arterioscler Thromb Vasc Biol 34: 2394-2403, 2014.

22. Leiva A, Verdejo H, Benitez ML, Martinez A, Busso D and Rigotti A: Mechanisms regulating hepatic SR-BI expression and their impact on HDL metabolism. Atherosclerosis 217: 299-307, 2011

23. Kozarsky KF, Donahee MH, Glick JM, Krieger M and Rader DJ: Gene transfer and hepatic overexpression of the HDL receptor SR-BI reduces atherosclerosis in the cholesterol-fed LDL receptor-deficient mouse. Arterioscler Thromb Vasc Biol 20: 721-727, 2000.

24. Huby T, Doucet C, Dachet C, Ouzilleau B, Ueda Y, Afzal V, Rubin E, Chapman MJ and Lesnik P: Knockdown expression and hepatic deficiency reveal an atheroprotective role for SR-BI in liver and peripheral tissues. J Clin Invest 116: 2767-2776, 2006

25. Van Eck M,Hoekstra M, Out R, Bos IS, Kruijt JK, Hildebrand RB and Van Berkel TJ: Scavenger receptor BI facilitates the metabolism of VLDL lipoproteins in vivo. J Lipid Res 49: 136-146, 2008

26. Yang XP, Amar MJ, Vaisman B, Bocharov AV, Vishnyakova TG, Freeman LA, Kurlander RJ, Patterson AP, Becker LC and Remaley AT: Scavenger receptor-BI is a receptor for lipoprotein(a). J Lipid Res 54: 2450-2457, 2013.

27. Yang X, Sethi A, Yanek LR, Knapper C, Nordestgaard BG Tybjærg-Hansen A, Becker DM, Mathias RA, Remaley AT and Becker LC: SCARB1 gene variants are associated with the phenotype of combined high high-density lipoprotein cholesterol and high lipoprotein (a). Circ Cardiovasc Genet 9: 408-418, 2016

28. Saddar S, Mineo C and Shaul PW: Signaling by the high-affinity HDL receptor scavenger receptor B type I. Arterioscler Thromb Vasc Biol 30: 144-150, 2010.

29. Rohrer L, Ohnsorg PM, Lehner M, Landolt F, Rinninger F and von Eckardstein A: High-density lipoprotein transport through aortic endothelial cells involves scavenger receptor BI and ATP-binding cassette transporter G1. Circ Res 104: 1142-1150, 2009.

30. Armstrong SM, Sugiyama MG, Fung KY, Gao Y, Wang C, Levy AS, Azizi P, Roufaiel M, Zhu SN, Neculai D, et al: A novel assay uncovers an unexpected role for SR-BI in LDL transcytosis. Cardiovasc Res 108: 268-277, 2015.

31. Seetharam D, Mineo C, Gormley AK, Gibson LL, Vongpatanasin W, Chambliss KL, Hahner LD, Cummings ML, Kitchens RL, Marcel YL, et al: High-density lipoprotein promotes endothelial cell migration and reendothelialization via scavenger receptor-B type I. Circ Res 98: 63-72, 2006.

32. Mineo C and Shaul PW: Role of high-density lipoprotein and scavenger receptor B type I in the promotion of endothelial repair. Trends Cardiovasc Med 17: 156-161, 2007.

33. Zhu W, Saddar S, Seetharam D, Chambliss KL, Longoria C, Silver DL, Yuhanna IS, Shaul PW and Mineo C: The scavenger receptor class B type I adaptor protein PDZK1 maintains endothelial monolayer integrity. Circ Res 102: 480-487, 2008.

34. Vaisman BL, Vishnyakova TG, Freeman LA, Amar MJ, Demosky SJ, Liu C, Stonik JA, Sampson ML, Pryor M, Bocharov AV, et al: Endothelial expression of scavenger receptor class B, type I protects against development of atherosclerosis in mice. Biomed Res Int 2015: 607120, 2015.

35. Fung KY, Wang C, Nyegaard S, Heit B, Fairn GD and Lee WL: SR-BI mediated transcytosis of HDL in brain microvascular endothelial cells is independent of caveolin, clathrin, and PDZK1. Front Physiol 8: 841, 2017.

36. Gadea $G$ and Blangy A: Dock-family exchange factors in cell migration and disease. Eur J Cell Biol 93: 466-477, 2014
37. Fluiter K, van der Westhuijzen DR and van Berkel TJ: In vivo regulation of scavenger receptor $\mathrm{BI}$ and the selective uptake of high density lipoprotein cholesteryl esters in rat liver parenchymal and kupffer cells. J Biol Chem 273: 8434-8438, 1998

38. Yancey PG, de la Llera-Moya M, Swarnakar S, Monzo P, Klein SM, Connelly MA, Johnson WJ, Williams DL and Rothblat GH: High density lipoprotein phospholipid composition is a major determinant of the bi-directional flux and net movement of cellular free cholesterol mediated by scavenger receptor BI. J Biol Chem 275: 36596-36604, 2000.

39. Matveev S, van der Westhuyzen DR and Smart EJ: Co-expression of scavenger receptor-BI and caveolin-1 is associated with enhanced selective cholesteryl ester uptake in THP-1 macrophages. J Lipid Res 40: 1647-1654, 1999.

40. Ji Y, Jian B, Wang N, Sun Y, Moya ML, Phillips MC, Rothblat GH, Swaney JB and Tall AR: Scavenger receptor BI promotes high density lipoprotein-mediated cellular cholesterol efflux. J Biol Chem 272: 20982-20985, 1997.

41. Mineo C: Lipoprotein receptor signalling in atherosclerosis. Cardiovasc Res 116: 1254-1274, 2020.

42. Song GJ, Kim SM, Park KH, Kim J, Choi I and Cho KH: SR-BI mediates high density lipoprotein (HDL)-induced anti-inflammatory effect in macrophages. Biochem Biophys Res Commun 457: 112-118, 2015.

43. Tao H, Yancey PG, Babaev VR, Blakemore JL, Zhang Y, Ding L, Fazio S and Linton MF: Macrophage SR-BI mediates efferocytosis via $\mathrm{Src} / \mathrm{PI} 3 \mathrm{~K} / \mathrm{Racl}$ signaling and reduces atherosclerotic lesion necrosis. J Lipid Res 56: 1449-1460, 2015.

44. Karackattu SL, Picard MH and Krieger M: Lymphocytes are not required for the rapid onset of coronary heart disease in scavenger receptor class B type I/apolipoprotein E double knockout mice. Arterioscler Thromb Vasc Biol 25: 803-808, 2005.

45. Van Eck M, Bos IS, Hildebrand RB, Van Rij BT and Van Berkel TJ: Dual role for scavenger receptor class B, type I on bone marrow-derived cells in atherosclerotic lesion development. Am J Pathol 165: 785-794, 2004.

46. Pei Y, Chen X, Aboutouk D, Fuller MT, Dadoo O, Yu P, White EJ, Igdoura SA and Trigatti BL: SR-BI in bone marrow derived cells protects mice from diet induced coronary artery atherosclerosis and myocardial infarction. PLoS One 8: e72492, 2013.

47. Badeau RM, Metso J, Wähälä K, Tikkanen MJ and Jauhiainen M: Human macrophage cholesterol efflux potential is enhanced by HDL-associated 17beta-estradiol fatty acyl esters. J Steroid Biochem Mol Biol 116: 44-49, 2009.

48. Vergeer M, Korporaal SJ, Franssen R, Meurs I, Out R, Hovingh GK, Hoekstra M, Sierts JA, Dallinga-Thie GM, Motazacker MM, et al: Genetic variant of the scavenger receptor BI in humans. N Engl J Med 364: 136-145, 2011.

49. Lindemann S, Krämer B, Daub K, Stellos K and Gawaz M: Molecular pathways used by platelets to initiate and accelerate atherogenesis. Curr Opin Lipidol 18: 566-573, 2007.

50. Olas B: Biochemistry of blood platelet activation and the beneficial role of plant oils in cardiovascular diseases. Adv Clin Chem 95: 219-243, 2020

51. Valiyaveettil M, Kar N, Ashraf MZ, Byzova TV, Febbraio M and Podrez EA: Oxidized high-density lipoprotein inhibits platelet activation and aggregation via scavenger receptor BI. Blood 111: 1962-1971, 2008

52. Brodde MF, Korporaal SJ, Herminghaus G, Fobker M, Van Berkel TJ, Tietge UJ, Robenek H, Van Eck M, Kehrel BE and Nofer JR: Native high-density lipoproteins inhibit platelet activation via scavenger receptor BI: Role of negatively charged phospholipids. Atherosclerosis 215: 374-382, 2011.

53. Brill A, Yesilaltay A, De Meyer SF, Kisucka J, Fuchs TA, Kocher O, Krieger M and Wagner DD: Extrahepatic high-density lipoprotein receptor SR-BI and apoA-I protect against deep vein thrombosis in mice. Arterioscler Thromb Vasc Biol 32: 1841-1847, 2012.

54. Korporaal SJ, Meurs I, Hauer AD, Hildebrand RB, Hoekstra M, Cate HT, Praticò D, Akkerman JW, Van Berkel TJ, Kuiper J and Van Eck M: Deletion of the high-density lipoprotein receptor scavenger receptor BI in mice modulates thrombosis susceptibility and indirectly affects platelet function by elevation of plasma free cholesterol. Arterioscler Thromb Vasc Biol 31: 34-42, 2011.

55. OuweneelAB,HoekstraM,vanderWelEJ,SchaftenaarFH,SnipOSC, Hassan J, Korporaal SJA and Van Eck M: Hypercholesterolemia impairs megakaryopoiesis and platelet production in scavenger receptor BI knockout mice. Atherosclerosis 282: 176-182, 2019. 